\title{
Posição prona no tratamento da insuficiência respiratória aguda na COVID-19*
}

\author{
Prone position in the treatment of acute respiratory failure due to COVID-19*
}

\begin{abstract}
BORGES, Daniel Lago ${ }^{1}$; RAPELLO, Gabriel Victor Guimarães²; DEPONTI, Gracieli Nadalon³ ANDRADE, Flávio Maciel Dias de ; em nome do Comitê COVID-19 da ASSOBRAFIR.
\end{abstract}

\section{Resumo}

Este documento tem por objetivo trazer informações sobre a utilização da posição prona no tratamento da insuficiência respiratória aguda (IRpA) secundária à COVID-19, bem como alertar para contraindicações e possíveis complicações do seu uso no ambiente da terapia intensiva. Embora a posição prona seja um recurso terapêutico que pode melhorar o processo de oxigenação de pacientes com SDRA, sugerimos cautela na indicação deste posicionamento durante a pandemia do COVID-19, especialmente em UTIs improvisadas, com time reduzido e não treinado. Nesse sentido, reforçamos a necessidade de treinamento dos fisioterapeutas e colegas das equipes multiprofissionais que atuam nas UTIs para que possam empregar com segurança esse recurso, sempre que houver necessidade e condições de segurança para sua realização.

Palavras-chave: Fisioterapia; Padrão de Cuidado; COVID-19.

\section{Abstract}

This document aims to provide information on the use of prone position in the treatment of Acute Respiratory Failure (ARF) secondary to COVID-19, as well as to highlight contraindications and possible complications of its use in the intensive care unit. Although prone position has been shown to be an effective therapeutic strategy to improve oxygenation in patients with Acute Respiratory Distress Syndrome (ARDS), we recommend caution when indicating this intervention for COVID-19 patients, especially in improvised ICUs with reduced

\footnotetext{
* Revisado por membros do Comitê COVID-19 da ASSOBRAFIR, nomeado por meio do memorando Nº 003/2020. Esta publicação é uma atualização da Comunicação Oficial "Posição prona no tratamento da insuficiência respiratória aguda na COVID-19", chancelada pelo Comitê COVID-19 da ASSOBRAFIR, originalmente escrita pelos mesmos autores e divulgada em 26/03/2020 no endereço eletrônico https://assobrafir.com.br/covid-19-posicao-prona/.

1 Hospital Universitário da Universidade Federal do Maranhão (HUUFMA), São Luís, Maranhão, Brasil. Email: dlagofisio83@hotmail.com. DLB - https://orcid.org/0000-0003-4082-527X

2 Hospital CASSEMS, Campo Grande, Mato Grosso do Sul, Brasil. GVGR - https://orcid.org/000-0002-5219-851X

3 Serviço de Fisioterapia do Hospital de Clínicas de Porto Alegre (HCPA), Porto Alegre, Rio Grande do Sul, Brasil.

${ }^{4}$ Universidade Católica de Pernambuco, Recife, Pernambuco, Brasil. FMDA - https://orcid.org/0000-0001-9571-6551
} 
and untrained staff. In this sense, we reinforce the need for a proper training of physiotherapists and colleagues from the multidisciplinary team working in ICUs so that they can safely employ this intervention, whenever there is a need and the patient's health condition allows.

Keywords: Physiotherapy; Prone position; COVID-19.

\section{Objetivo}

O objetivo do presente posicionamento é fornecer informações sobre a utilização da posição prona no tratamento da insuficiência respiratória aguda (IRpA) secundária à COVID-19, bem como alertar para contraindicações e possíveis complicações do seu uso no ambiente da terapia intensiva.

\section{Contextualização}

A doença causada pelo novo coronavírus (2019-nCoV, ou em inglês: Coronavirus Disease 2019; COVID-19), é uma infecção que compromete o trato respiratório, que foi identificada inicialmente em Wuhan, China, em dezembro de 2019 ${ }^{1,2,3}$.

Enquanto a maioria dos pacientes infectados não desenvolve complicações ou apresenta apenas sintomas leves, aproximadamente $14 \%$ evoluem para um estágio mais grave que requer hospitalização, suporte de oxigênio e, por vezes, ventilação mecânica (VM). Destes, de 5\% a 26\% dos casos necessitam internação em unidade de terapia intensiva (UTI) ${ }^{1,2}$. A COVID-19 pode desencadear complicações como sepse (59\%), insuficiência renal aguda (15\% a 29\%), disfunção cardíaca aguda (17\% a 23\%) e síndrome do desconforto respiratório agudo (SDRA) (31\% a 67\%) $)^{2,4}$.

No tratamento de pacientes com SDRA, uma estratégia que se destaca é a posição prona. Esta estratégia consiste em posicionar o paciente em decúbito ventral, o que deve resultar em distribuição mais uniforme do estresse e da tensão pulmonar, melhora da relação ventilação/perfusão, da mecânica pulmonar e da parede torácica ${ }^{5}$, contribuindo para redução da duração da VM e da taxa de mortalidade avaliada em um seguimento de 28 e 90 dias $^{6}$. Dados de UTI's da China e Itália demonstram o uso da posição prona como recurso terapêutico em 10 a $30 \%$ dos pacientes internados em $\mathrm{UTI}^{7}$.

\section{Indicação da posição prona}

A posição prona deve ser utilizada precocemente (até nas primeiras 48 horas, de preferência nas primeiras 24 horas), em pacientes que apresentem SDRA e alteração grave da troca gasosa, caracterizada por uma relação entre pressão parcial de oxigênio arterial - $\mathrm{PaO}_{2}$ e fração inspirada de oxigênio - $\mathrm{FiO}_{2}\left(\mathrm{PaO}_{2} / \mathrm{FiO}_{2}\right)$ inferior a $150 \mathrm{mmHg}$. Quando adotada, deve ser mantida por pelo menos 16 horas (podendo atingir 20 horas), antes de retornar o paciente para posição supina ${ }^{6}$.

Recentemente Gattinoni et al. ${ }^{8,9}$ publicaram editoriais indicando acometimento pulmonar diferenciado dos pacientes com SARS-COV-2 e sugeriram a divisão em 2 fenótipos "L" ou "H", ou ainda em tipo "1" e tipo "2". Por análises tomográficas eles indicam que a posição prona, assim como todas as estratégias de ventilação para SDRA, é um recurso que deve ser empregado em pacientes com alterações do tipo 2, ou seja, que apresentam alto potencial de recrutabilidade. Já em pacientes do tipo 1 a posição prona pode ser considerada mais como uma estratégia de resgate para facilitar a redistribuição do fluxo sanguíneo e a abertura de áreas colapsadas, porém com pouco benefício nestes pacientes com complacência pulmonar normal ou alta. 


\section{Contraindicações para a realização da manobra de prona}

As principais contraindicações para realização da posição prona são $0^{5,10}$ :

Absolutas

- Arritmias graves agudas

- Fraturas pélvicas

- Pressão intracraniana não monitorada ou significativamente aumentada

- Fraturas vertebrais instáveis

- Esternotomia recente

- Peritoneostomia

Relativas

- Difícil manejo das vias aére

- Cirurgia traqueal ou esternotomia nos últimos 15 dias

- Traqueostomia há menos de 24 horas

- Dreno torácico anterior com vazamento de ar

- Trauma ou ferimentos faciais graves ou cirurgia facial nos últimos 15 dias

- Cirurgia oftalmológica ou pressão intraocular aumentada

- Instabilidade hemodinâmica ou parada cardiorrespiratória recente

- Marcapasso cardíaco inserido nos últimos 2 dias

- Dispositivo de assistência ventricular

- Balão intra-aórtico

- Trombose venosa profunda tratada por menos de 2 dias

- Hemoptise maciça ou hemorragia pulmonar que requer um procedimento cirúrgico ou intervencionista imediata

- Diálise contínua

- Lesões graves da parede torácica ou fraturas de costelas

- Cirurgia cardiotorácica recente ou tórax instável

- Politrauma com fraturas não estabilizadas

- Gestação

- Cirurgia abdominal recente ou formação de estoma

- Cifoescoliose

- Osteoartrite ou artrite reumatoide avançadas

- Peso corpóreo superior a $135 \mathrm{Kg}$

- Pressão intra-abdominal $>20 \mathrm{mmHg}$

\section{Critérios de sucesso da manobra de prona}

Após 1 (uma) hora em posição prona, uma gasometria deve ser realizada para avaliar se o paciente responde ou não a esta estratégia. Caso seja considerado como respondedor (aumento de 20 mmHg na relação $\mathrm{PaO}_{2} / \mathrm{FiO}_{2}$ ou de $10 \mathrm{mmHg}$ na $\mathrm{PaO}_{2}$ ), o posicionamento deve ser mantido. Atentar que alguns pacientes são respondedores lentos (25\%), levando em torno de 6 horas para indicar resposta favorável à posição prona, portanto sugere-se que a avaliação seja repetida em 6 horas. Do contrário, retorna-se o paciente à posição supina. Sugere-se que esta avaliação seja repetida a cada 6 (seis) horas. Não havendo mais sinais de resposta, o paciente deve ser retornado à posição supina ${ }^{10}$. 


\section{Interrupção da manobra de prona e retorno à posição supina}

A presença de sinais de sofrimento cutâneo também pode indicar a necessidade de interrupção do posicionamento prono.

O posicionamento prono também deve ser interrompido em casos de ocorrência de complicações, tais como ${ }^{6}$ :

- extubação não programada

- obstrução do tubo endotraqueal

- hemoptise

- saturação periférica de oxigênio $\left(\mathrm{SpO}_{2}\right)<85 \%$ ou $\mathrm{PaO}_{2}<55 \mathrm{mmHg}$ por mais de 5 minutos, com $\mathrm{FiO}_{2}=100 \%$

- parada cardiorrespiratória

- frequência cardíaca (FC) $<30 \mathrm{bpm}$ por mais de 1 (um) minuto

- pressão arterial sistólica $<60 \mathrm{mmHg}$ por mais de 5 (cinco) minutos

- qualquer outro motivo potencialmente fatal

A gasometria arterial dos pacientes considerados respondedores deve ser reavaliada 4 (quatro) horas após o retorno à posição supina e a presença de uma relação $\mathrm{PaO}_{2} / \mathrm{FiO}_{2}<150 \mathrm{mmHg}$ é indicativa da necessidade de realização de um novo ciclo de prona. Observando-se piora da hipoxemia durante a posição supina em um intervalo de tempo menor que 4 (quatro) horas, a utilização de manobra de recrutamento deve ser considerada para melhora da hipoxemia e aumento do tempo para realização de um novo ciclo de prona. Havendo uma relação $\mathrm{PaO}_{2} / \mathrm{FiO}_{2}>150 \mathrm{mmHg}$ após 4 (quatro) horas de posição supina, recomenda-se a suspensão dos ciclos de prona e manutenção do paciente em supino ${ }^{10}$.

\section{Complicações da manobra de prona}

O posicionamento em prona é um procedimento que não está isento de riscos e as principais complicações associadas estão listadas abaixo ${ }^{11}$ :

- Edema (facial, vias aéreas, membros, tórax)

- Lesões por pressão

- Hemorragia conjuntival

- Compressão de nervos e vasos retinianos

- Obstrução, pinçamento ou deslocamento (intubação seletiva ou extubação não-programada) do tubo endotraqueal

- Dificuldade para aspiração das vias aéreas

- Hipotensão transitória ou queda da saturação periférica de oxigênio

- Piora das trocas gasosas

- Pneumotórax

- Obstrução ou pinçamento de drenos torácicos ou cateteres vasculares

- Eventos cardíacos

- Deslocamento inadvertido do cateter de Swan-Ganz

- Trombose venosa profunda

- Deslocamento de sonda vesical ou nasoentérica

- Intolerância à nutrição enteral; vômito; complicações alimentares

- Necessidade de maior sedação ou bloqueio neuromuscular

- Dificuldade em instituir ressuscitação cardiopulmonar. 


\section{Fatores determinantes de sucesso da manobra}

Um dos pontos importantes para a realização da manobra de forma segura e que indicam maiores chances de sucesso é a realização por equipe experiente e devidamente treinada. Para isso, sugere-se que os centros que realizam posicionamento prono disponibilizem treinamentos e formação continuada para seus colaboradores ${ }^{12}$. Outro fator importante para o sucesso da manobra é o número adequado de profissionais. Para execução da manobra de posicionamento em prona sugere-se a participação de 3 (três) a 5 (cinco) profissionais. Recomenda-se que a equipe seja treinada e sugere-se que assistam a vídeos de execução da manobra. Seguem alguns links ${ }^{10}$ :

- PROSEVA (3 pessoas): http://www.youtube.com/watch?v=E 6jT9R7WJs

- William Harvey Hospital (5 pessoas): http://www.youtube.com/watch?v=Hd5o4ldp3c0

- Hospital de Clínicas de Porto Alegre (HCPA): https://drive.google.com/file/d/14YFYSZXDadPdfLZR1oBz16OCmIJgz16v/view https://drive.google.com/file/d/1SHE8F8tnvj]lLyQBTg0tYHXHrBtjeyL6/view

\section{Realização da Manobra de Prona}

Antes de realizar a manobra, alguns cuidados devem ser observados ${ }^{11-13}$ :

- pausar dieta e abrir sonda nasoentérica 2 (duas) horas antes do procedimento caso não seja possível, avaliar se o paciente estava recebendo dieta e caso sim, realizar a aspiração do conteúdo gástrico);

- providenciar coxins para apoio de tórax e pelve, e se possível para face, punho e região anterior das pernas (tarefa importante desempenhada pelo fisioterapeuta, que avaliará a composição corporal do paciente e indicará os coxins mais adequados para manter o abdômen do paciente livre e com isto permitir a melhora da ventilação pulmonar);

- aproximar carro de parada cardiorrespiratória, caixa de intubação e testar material de aspiração;

- realizar cuidados oculares e para pele;

- revisar fixação dos dispositivos invasivos, curativos e via aérea artificial (aspirar vias aéreas, verificar fixação, medir pressão do balonete do tubo endotraqueal e registrar comissura labial);

- pausar hemodiálise contínua (recircular e heparinizar cateter) caso em uso;

- pré-oxigenar com $\mathrm{FiO}_{2}=100 \%$ por 10 minutos;

- ajustar analgosedação e avaliar a necessidade de bloqueio neuromuscular.

Para a confecção dos coxins o fisioterapeuta deve avaliar a composição corporal do paciente, principalmente a distância do tórax (ombro a ombro) e quadril (cristas ilíacas) e confeccionar o coxim no tamanho e altura adequados para manter o abdômen livre. Recomendamos seguir o Manual do uso e confecção de coxins para manobra de prona elaborado pelo Grupo de Prona da UTI do $\mathrm{HCPA}^{14}$.

Alguns cuidados devem ser tomados no momento da realização do giro:

- reunir a equipe que realizará o giro (3 a 5 pessoas);

- desconectar e fechar sonda nasoenteral, clampear sondas, drenos e posicioná-los entre as pernas e braços; 
- colocar a cabeceira em posição plana e alinhar os membros;

- pausar infusões e desconectar cateteres;

- especialmente em pacientes com risco de formação de aerossol, revisar atentamente todas as conexões do circuito da VM para evitar desconexões (risco elevado de contaminação da equipe);

- posicionar coxins sobre tórax e pelve do paciente e colocar lençol superior para formação do envelope;

- realizar a técnica em envelope, dividida em 3 (três) momentos: deslocamento para o lado contrário ao ventilador (após esse movimento, posiciona-se os coxins), lateralização e posição prona.

Após o procedimento, deve-se checar o posicionamento do tubo endotraqueal pela ausculta pulmonar e comissura labial, além de confirmar a pressão do balonete do tubo. A cabeceira da cama deve estar posicionada em Trendelemburg reverso, para reduzir o risco de aspiração $\left(20^{\circ}\right)$.

Os membros superiores devem ser posicionados em posição de nadador (um braço fletido para cima e outro estendido para baixo, com rosto virado para o braço fletido), com alternância a cada 2 (duas) horas, evitando a lesão do plexo braquial (Figura 1) ${ }^{12}$. Este procedimento deve ser realizado por no mínimo 2 profissionais para ser mais seguro. Porém, em pacientes com COVID-19, o tempo para troca de posição nadador pode ser estendido já que os profissionais necessitam de paramentação e EPIS adequados e há risco de desconexão do circuito. Sugere-se então que a troca da posição de nadador seja realizada a cada 4 a 6 horas, conferindo sempre as conexões do circuito de VM. Atentar para o risco aumentado de piora do edema e lesão de pele na face no paciente. Os eletrodos do eletrocardiograma devem ser posicionados no dorso.

Sugere-se a utilização de placas hidrocoloides para proteção da testa, face, joelhos e ombros (e demais locais com saliências ósseas), além da colocação de travesseiros na frente do paciente, distribuídos de modo a aliviar os pontos de apoios anatômicos principais ${ }^{13}$.

Figura 1 | Posição de nadador

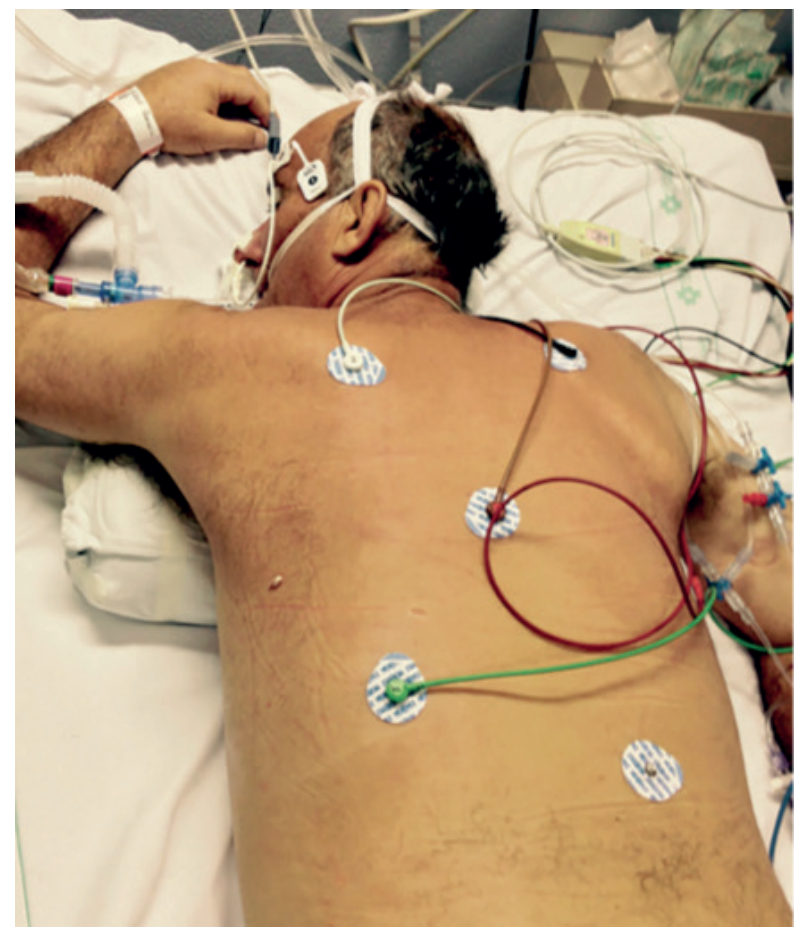




\section{Considerações sobre posicionamento do paciente e realização da manobra}

Em serviços onde não estejam disponíveis os coxins de gel para a face (tipo arandela), pode se confeccionar um coxim com compressas ou qualquer material menos denso (pedaços de piramidal algodão etc) e atadura em forma de arandela para posicionar a face deixando o olho e a orelha do paciente liberados.

O Fisioterapeuta deve conferir todo o posicionamento do paciente paraidentificardesalinhamentos corporais e possíveis áreas de lesão de pele e osteomuscular, realizando as devidas correções.

Um recurso que pode ser útil é a implementação de ferramentas para segurança da manobra, do paciente e da equipe. Sugere-se a utilização de um Check-list para a colocação do paciente em prona e em supina.

Um modelo de Check-list pode ser acessado no link:

https://drive.google.com/drive/folders/13COGkeeK eNZwg85YDt y7PPKf83pzt

A seguir apresenta-se um fluxograma para utilização da posição prona (Figura 2).

Figura 2 | Fluxograma de protocolo para posição prona. Adaptado de Oliveira e cols. ${ }^{12}$

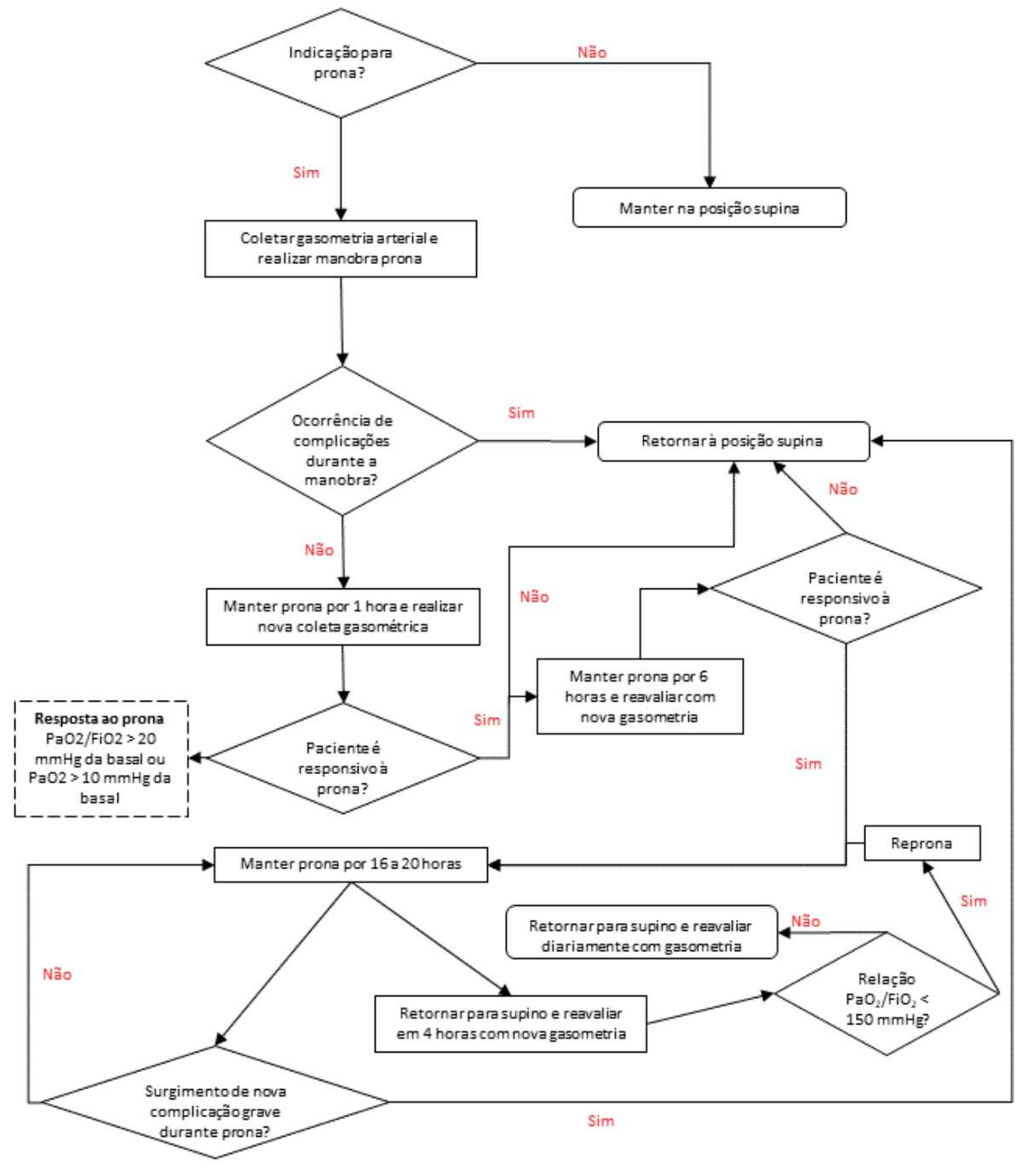




\section{Realização de posição prona em pacientes em ventilação espontânea (suplementação de 02, Cateter Nasal de Alto Fluxo - CNAF ou Ventilação Não Invasiva - VNI) - Auto-Prona ou Prona Ativa}

Até o presente momento não existem evidências suficientes para recomendar o uso rotineiro da posição prona em pacientes em ventilação espontânea (oxigenoterapia, CNAF ou VNI) ${ }^{15-16}$.

Recentemente foram publicadas duas cartas e um estudo observacional com amostra por conveniência em pacientes com COVID-19 sugerindo a utilização de posição prona em pacientes não intubados com hipoxemia sustentada apesar da suplementação de $\mathrm{O}_{2}$ ou uso de VNI e CNAF ${ }^{17-19}$. Como já demonstrado em estudo prévios ${ }^{20}$, a posição prona em pacientes não intubados é capaz de melhorar a $\mathrm{SpO}_{2}$ e aumentar a $\mathrm{PaO}_{2}$ na maioria dos pacientes enquanto permanecem neste decúbito. Alguns pacientes apresentam melhora sustentada $\mathrm{PaO}_{2}$ mesmo após retorno a posição supina e em alguns pacientes esta terapêutica foi eficaz para reduzir necessidade de IOT e VM invasiva. Porém ainda não são trabalhos suficientes para determinar o perfil de pacientes que se beneficiariam desta manobra, por quanto tempo devem ser mantidos em prona, quais os possíveis riscos e benefícios, nem se há real impacto sobre redução de mortalidade nos pacientes com COVID-19. Alguns pontos importantes que devem ser observados é que quando se avalia realizar a prona em pacientes não intubados, o paciente deve ser capaz de auxiliar ou realizar de forma ativa a manobra, portanto deve estar alerta, tranquilo e colaborativo, além de ter força muscular e apresentar certa estabilidade ventilatória, ou seja, não apresentar sinais clínicos de necessidade imediata de IOT. Também há necessidade de monitorização rigorosa com avaliação dos sinais vitais $\left(\mathrm{SpO}_{2}\right.$ que deve apresentar melhora em até 5 a 10 minutos após o posicionamento e estabilidade nos demais sinais vitais - frequência cardíaca, frequência respiratória e pressão arterial) e tolerância do paciente ao posicionamento.

Além disto, alguns experts indicam o uso da posição prona em casos selecionados, ou seja, pacientes que se mantém hipoxêmicos mesmo com otimização da oxigenoterapia, principalmente associada à uso de CNAF ou VNI e em situações que NÃO existam aparelhos de VM invasiva disponíveis para todos os pacientes. Estas indicações são baseadas em evidências anedóticas e dados de observação direta em outros UTIs do mundo relatando viabilidade de realização da manobra e melhora temporária da oxigenação em pacientes com COVID-19 ${ }^{21}$.

\section{Considerações Finais}

Embora a posição prona seja um recurso terapêutico que pode melhorar o processo de oxigenação de pacientes com SDRA, sugerimos cautela na indicação deste posicionamento durante a pandemia do COVID-19, especialmente em UTIs improvisadas, com time reduzido e não treinado. Nesse sentido, reforçamos a necessidade de treinamento dos fisioterapeutas e colegas das equipes multiprofissionais que atuam nas UTIs para que possam empregar com segurança esse recurso, sempre que houver necessidade e condições de segurança para sua realização. 


\section{Referências}

1. Epidemiology Working Group for NCIP Epidemic Response, Chinese Center for Disease Control and Prevention. [The epidemiological characteristics of an outbreak of 2019 novel coronavirus diseases (COVID-19) in China]. Zhonghua Liu Xing Bing Xue Za Zhi. 2020 Feb 10;41(2):145-151. doi: 10.3760/cma.j.issn.0254-6450.2020.02.003. Chinese.

2. Zhou F, Yu T, Du R, Fan G, Liu Y, Liu Z, et al. Clinical course and risk factors for mortality of adult inpatients with COVID-19 in Wuhan, China: a retrospective cohort study. Lancet. 2020 Mar 28;395(10229):1054-1062. doi: 10.1016/S0140-6736(20)30566-3. Epub 2020 Mar 11.

3. Matte DL, Andrade FMD, Martins JA, Martinez BP, Karsten M. O fisioterapeuta e sua relação com o novo betacoronavirus 2019 (2019-nCoV): comunicação oficial da ASSOBRAFIR [Internet]. São Paulo: ASSOBRAFIR; 2020. Available from: https://assobrafir.com.br/assobrafir betacoronavirus2019/2020.

4. Yang X, Yu Y, Xu J, Shu H, Xia J, Liu H, et al. Clinical course and outcomes of critically ill patients with SARS-CoV-2 pneumonia in Wuhan, China: a single-centered, retrospective, observational study. Lancet Respir Med. 2020 May;8(5):475-481. doi: 10.1016/S2213-2600(20)30079-5. Epub 2020 Feb 24.

5. Koulouras V, Papathanakos G, Papathanasiou A, Nakos G. Efficacy of prone position in acute respiratory distress syndrome patients: A pathophysiology-based review. World J Crit Care Med. 2016 May 4;5(2):121-36. doi: 10.5492/wjccm.v5.i2.121.

6. Guérin C, Reignier J, Richard JC, Beuret P, Gacouin A, Boulain T, et al. Prone positioning in severe acute respiratory distress syndrome. N Engl J Med. 2013 Jun 6;368(23):2159-68. doi: 10.1056/ NEJMoa1214103. Epub 2013 May 20.

7. Grasselli G, Zangrillo A, Zanella A, Antoneli M, Cabrini L, Castelli A, et al. Baseline Characteristics and Outcomes of 1591 Patients Infected With SARS-CoV-2 Admitted to ICUs of the Lombardy Region, Italy. JAMA. 2020 Apr 6;323(16):1574-1581. doi: 10.1001/jama.2020.5394. Online ahead of print.

8. Gattinoni L, Chiumello D, Caironi P, Busana M, Romitti F, Brazzi L et al. COVID-19 pneumonia: different respiratory treatment for different phenotypes? Intensive Care Med. 2020 Jun;46(6):10991102. doi: 10.1007/s00134-020-06033-2. Epub 2020 Apr 14.

9. Gattinoni L, Chiumello D, Rossi S. COVID-19 pneumonia: ARDS or not? Crit Care. 2020 Apr 16;24(1):154. doi: 10.1186/s13054-020-02880-z.

10. Barbas CSV, Ísola AM, Farias AMC, Cavalcanti AB, Gama AMC, Duarte ACM, et al. [Brazilian recommendations of mechanical ventilation 2013. Part I]. Rev Bras Ter Intensiva. 2014 Apr-Jun ;26(2): 89-121. doi: 10.5935/0103-507x.20140017. Portuguese.

11. Dirkes S, Dickinson S, Havey R, O’Brien D. Prone positioning: Is it safe and effective? Crit Care Nurs Q. 2012 Jan-Mar;35(1):64-75. doi: 10.1097/CNQ.0b013e31823b20c6.

12. Oliveira VM, Piekala DM, Deponti GN, Batista DCR, Minossi SD, Chisté M et al. Safe prone checklist: Construction and implementation of a tool for performing the prone maneuver. Rev Bras Ter Intensiva. Apr-Jun 2017;29(2):131-141. doi: 10.5935/0103-507X.20170023.

13. Oliveira VM, Weschenfelder ME, Deponti G, Condessa R, Loss SH, Bairros PM, et al. Good practices for prone positioning at the bedside: Construction of a care protocol. Rev Assoc Med Bras (1992). May-Jun 2016;62(3):287-93. doi: 10.1590/1806-9282.62.03.287. 
14. Grupo de Prona da UTI do Hospital de Clínicas de Porto Alegre. Manual do uso e confecção de coxins para manobra de prona. Porto Alegre: HCPA; 2019.

15. Ding L, Wang L, Ma W, He H. Efficacy and safety of early prone positioning combined with HFNC or NIV in moderate to severe ARDS: a multi-center prospective cohort study. Crit Care. 2020 Jan 30;24(1):28. doi: 10.1186/s13054-020-2738-5.

16. Pérez-Nieto OR, Guerrero-Gutiérrez MA, Deloya-Tomas E, Namendys-Silva SA. Prone positioning combined with high-flow nasal cannula in severe noninfectious ARDS. Crit Care. 2020 Mar 23;24(1):114. doi: 10.1186/s13054-020-2821-y.

17. Elharrar X, Trigui Y, Dols AM, Touchon F, Martinez S, Prud'homme E, et al. Use of Prone Positioning in Nonintubated Patients With COVID-19 and Hypoxemic Acute Respiratory Failure. JAMA. 2020 May 15;323(22):2336-8. doi: 10.1001/jama.2020.8255. Online ahead of print.

18. Sartini C, Moreno Tresoldi M, Scarpellini P, Tettamanti A, Carcò F, Landoni G, et al. Respiratory Parameters in Patients With COVID-19 After Using Noninvasive Ventilation in the Prone Position Outside the Intensive Care Unit. JAMA. 2020 May 15;323(22):2338-2340. doi: 10.1001/ jama.2020.7861. Online ahead of print.

19. Caputo N, Strayer RJ, Levitan R. Early Self-Proning in Awake, Non-intubated Patients in the Emergency Department: A Single ED's Experience During the COVID-19 Pandemic. Acad Emerg Med. 2020 May;27(5):375-378. doi: 10.1111/acem.13994.

20. Scaravilli V, Grasselli G, Castagna L, Zanella A, Isgro S, Lucchini A, et al. Prone positioning improves oxygenation in spontaneously breathing nonintubated patients with hypoxemic acute respiratory failure: A retrospective study. J Crit Care. 2015 Dec;30(6):1390-4. doi: 10.1016/j. jcrc.2015.07.008. Epub 2015 Jul 16.

21. Pan C, Chen L, Lu C, Zhang W, Xia JA, Sklar MC, et al. Lung Recruitability in SARS-CoV-2 Associated Acute Respiratory Distress Syndrome: A Single-center, Observational Study. Am J Respir Crit Care Med. 2020 May 15;201(10):1294-1297. doi: 10.1164/rccm.202003-0527LE.

Submissão em: 28/05/2020

Aceite em: 15/07/2020 